\title{
Comparison of Two Radiographic Lumbar Spine Screening Protocols for the Detection of Abnormalities in Special Forces Candidates
}

\author{
Ori Wand, Alon Grossman, Alex Prokupetz, Amit Assa
}

The Israeli Air Force Aero Medical Center, Tel Hashomer, Israel.

Email: alon2206@012.net.il

Received August 15 ${ }^{\text {th }}, 2011$; revised September 26 ${ }^{\text {th }}, 2011$; accepted October $14^{\text {th }}, 2011$.

\begin{abstract}
Background: Medical screening for Special Forces (SF) has significant implications on operational competency of the soldiers. Two lumbar spine defects, spondylolysis and spondylolisthesis, are currently disqualifying from joining the SF in the Israeli Defense Force. Purpose: To evaluate the yield of four lumbar x-ray compared with two lumbar x-ray for the diagnosis of spondylolysis and spondylolisthesis. Design: Retrospective comparison of the diagnostic yield of the two modalities. Patient sample: Special forces unit candidates undergoing routine $x$-ray for the diagnosis of spinal defects. Outcome measures: The rate of diagnosis of spondylolysis and spondylolisthesis between the two groups. Methods: We retrospectively compared two lumbar spine radiographic screening protocols regarding the rate of diagnosis for disqualifying spinal defects in asymptomatic candidates for SF. All radiographs were obtained as part of a routine medical evaluation for SF. Results: 1026 candidates were examined with two lumbar spine radiographs (Anteroposterior (AP) and lateral). 338 candidates were examined with four lumbar radiographs (AP, lateral and oblique radiographs). Spondylolysis and spondylolisthesis were diagnosed at a significantly higher rate in the four-radiograph group than in the two-radiograph group $(2.66 \%, 1.77 \%$ vs $1.26 \%, 0.39 \%$ respectively, $P=0.003)$. Conclusions: Adding two oblique radiographs to the screening process of elite units candidates significantly increases the rate of diagnosis of spondylolysis and spondylolisthesis.
\end{abstract}

Keywords: Spondylolysis, Spondylolisthesis, Low Back Pain

\section{Introduction}

Medical evaluation is a crucial part of the screening process for combat units. This is particularly evident in Special Forces (SF) units in which the physical activity is more demanding and the weights that the soldiers have to carry are significantly greater. In addition, the operational characteristics of those units make a medically incapacitated soldier a critical problem. Low back pain (LBP) is a common complaint among military personnel with rates as high as $20 \%$ reported in the literature $[1,2]$. This is particularly evident in soldiers engaged in intense physical activity, such as SF units [3]. Low back pain is associated with both loss of work days and inability to complete the intense training process that is typical of SF units. Thus, there is a need for improved screening in order to disqualify candidates with pathologies which can facilitate severe LBP [4]. Spondylolysis and spondylolisthesis are associated with high rates of low back pain particularly in preadolescent and adolescent athletes [5] and therefore are considered disqualifying from SF units [4]. We aimed to detect the rate of these spinal abnormalities in asymptomatic SF units candidates by use of lumbar spine plain radiography and to compare the rate of positive findings using a two-radiograph protocol (Anteroposterior (AP) and lateral views) and a four-radiograph protocol (adding two oblique lumbar radiographs $-45^{\circ}$ right oblique and $45^{\circ}$ left oblique).

\section{Materials and Methods}

The study population consisted of all SF units candidates who underwent medical evaluation at the Israeli air force (IAF) aeromedical center between the years 1997-2007. All candidates were asymptomatic healthy men, aged 17 years - 20 years old, whose evaluation was performed following basic medical screening. History of low back pain and physical findings of significant spinal pathologies are disqualifying from joining SF and therefore subjects who were symptomatic did not reach our evaluation. 
In the years 1997-2005 all candidates underwent only two lumbar radiographs (AP and lateral) and in the years 2005-2007, two additional radiographs were added (2 obliques).

The diagnosis of all spine radiographs was documented in a computerized database. We retrospectively compared the radiographic protocol of the first group (two lumbar radiographs-AP and lateral), with the lumbar radiographic protocol of the second group (four lumbar radiographs_-AP, lateral and 2 obliques).

Chi-square test was used to compare the distribution of positive diagnoses (spondylolysis and spondylolisthesis) between the 2 groups. A p-value of $<0.05$ was considered statistically significant.

This study was approved by the ethics committee of the Medical Corps of the Israeli Defense Force.

\section{Results}

The group who underwent two radiographs included 1,026 candidates and the group who underwent 4 radiographs included 338 candidates. $25 \%$ percent of radiographs were interpreted by a single orthopedic surgeon and $75 \%$ by a single radiologist in both groups.

\subsection{Lumbar 2-View Radiography}

In 13 of 1026 candidates from the first group, spondylolysis of L5 vertebra was detected on lumbar AP and lateral radiographs (1.26\%). Another 4 candidates were diagnosed with spondylolysis and spondylolisthesis of L5 (0.39\%). A total of $1.65 \%$ of the group was disqualified from joining the Special Forces based on their lumbar radiographs. Additional findings, which had no implication on the medical screening process, are summarized in the table.

\subsection{Lumbar 4-View Radiography}

The second group consisted of 338 candidates. Spondylolysis of L5 was detected in 9 cases; another 6 candidates were diagnosed with spondylolysis and spondylolisthesis of L5 (see Table 1). Disqualification rates were $4.43 \%$ in this group, significantly higher than in the first group of candidates $(\mathrm{p}=0.003)$.

There were no diagnoses of spondylolysis or spondylolisthesis in vertebras other than L5.

\section{Discussion}

The unique operational and training demands of SF mandate strict medical evaluation as part of the screening process for candidates. The medical evaluation is performed in order to diagnose current medical conditions which are incompatible with the expected requirements of the service, as well as to reduce the possibility of such conditions in the future. Orthopedic problems, and specifically LBP, constitute a significant portion of the medically inadequate soldier.

In our screening process, a history of LBP is disqualifying from joining the SF. It is difficult to predict which recruits will develop significant LBP during military service, but spondylosis and spondylolisthesis may increase this risk.

Plain radiography is a readily-available modality to assess for spinal defects in asymptomatic subjects. About $20 \%$ of the spondylytic pars defects are seen only in lateral oblique views [6,7]. However, the iliac bones may obscure the images of the lower lumbar vertebrae where spondylolysis occurs more frequently. Also, plain radiographs are most useful when the plane of the radiological beam is aligned parallel to the plane of the defect. Saifuddin et al. used CT scans of spondylytic defects and found that only $32 \%$ of pars defects aligned within $15^{\circ}$ of the $45^{\circ}$ lateral oblique plain [6]. Thus, $45^{\circ}$ lateral oblique radiography may fail to detect a significant portion of the spondylytic defects, and its diagnostic value is unproven [6]. Although the negative predictive value of plain radiographs for the diagnosis of spondylolysis is quite low, it is considered quite specific.

Table 1. Comparison of lumbar radiographic results between the two study groups.

\begin{tabular}{ccc}
\hline Findings & 2-View & 4-View \\
Normal & $945(92.1)$ & $314(92.8)$ \\
Minimal Scoliosis & $32(3.1)$ & $6(2)$ \\
Spina Bifida Occulta & $22(2.1)$ & $2(0.6)$ \\
Schmorl' Nodes & $10(1)$ & $1(0.3)$ \\
Spondylolysis & $13(1.26)$ & $9(2.66)$ \\
Spondylolysis with Spondylolis- \\
thesis \\
Disqualifying Findings*
\end{tabular}

*All figures represent absolute number (percent). The 2-View group consists of candidates who underwent AP and Lateral lumbar radiographs. The 4-View group consists of candidates who underwent AP, Lateral and 2 Oblique lumbar radiographs. 
We use plain radiography rather than other imaging modalities due to logistic issues and high availability during the screening process. The prevalence of spondylolysis and spondylolisthesis observed in the 4-view group in our study $(4.43 \%)$ is consistent with the previously published prevalence of $4 \%$ - $6 \%$ [8].

The prognostic value of spinal defects in asymptomatic patients is controversial. In the work of Beutler et al., the natural history of spondylolysis and spondylolisthesis in a group of patients unselected for pain was benign. The clinical course was similar to the general population, and there appeared to be a slowing of the slip progression with each decade [8]. A similar incidence of sickness absenteeism due to LBP was found among police officers with spondylolysis and without it [9].

No difference in the incidence of LBP between soldiers with spondylolysis or without it was noted in the study by Libson et al. They did notice an association of pain with spondylolisthesis. Also, the pars defects were seen only in the oblique views in $18.7 \%$ of the cases [10]. A more recent study demonstrated increased frequency of spinal radiographic findings, including spondylolysis, in male army recruits complaining of LBP [11].

The incidence of LBP in rugby and football players who were diagnosed with spondylolysis before athletic participation was $72.5 \%-80.5 \%$ during 1 year of follow-up, compared to $32.1 \%$ - $44 \%$ in the group of players with normal lumbar radiographs $[12,13]$.

In a retrospective observational study, LBP accounted for nearly a quarter of the medical downgrading causes of soldiers in a British commando unit [14]. Eighteen percent of healthy male infantry recruits were diagnosed with overexertional back pain during the course of 14 weeks of training in another study [15].

The intensive activity of SF is significantly different from that of other military units and, of course, from that of other non-military professions. We believe that this unique physical activity may jeopardize soldiers with spinal defects for severe LBP. We found no studies addressing the incidence or the outcome of SF soldiers with spondylolysis and spondylolisthesis. We also acknowledge that normal spinal radiographs do not preclude the soldier from developing LBP in the future.

As this study was aimed at detection of spondylolysis and spondylolisthesis between the two modalities, no follow-up of the subjects was performed regarding the occurrence of symptoms. Such a study would shed more light on the association between radiologic findings and future occurrence of back symptoms in asymptomatic subjects.

\section{Conclusions}

Use of a four-radiograph method is more sensitive than a 2-radiograph method for the detection of spondylolysis and spondylolisthesis. Populations for which such screening is recommended may prefer to use a method with higher sensitivity in order to prevent potential injuries, symptoms and loss of personnel. Further research is required to correlate the occurrence of LBP symptoms with radiologic findings in asymptomatic subjects and to evaluate the use of other imaging modalities, such as CT scan, for this screening process.

\section{REFERENCES}

[1] T. Heir and P. Glomsaker, "Epidemiology of Musculoskeletal Injuries among Norwegian Conscripts Undergoing Basic Military Training," Scandinavian Journal of Medicine and Science in Sports, Vol. 6, No. 3, 1996, pp. 186191.

[2] H. Taanila, J. Suni, H. Pihlajamäki, V. M. Mattila, O. Ohrankämmen, P. Vuorinen and J. Parkkari, "Musculoskeletal Disorders in Physically Active Conscripts: A One Year Follow-Up Study in the Finnish Defense Forces," BMC Musculoskeletal Disorders, Vol. 10, 2009, p. 89. doi:10.1186/1471-2474-10-89

[3] K. R. Kaufman, S. Brodine and R. Shaffer, "MilitaryTraining Related Injuries: Surveillance, Research and Prevention," American Journal of Preventive Medicine, Vol. 18, No. 3, 2000, pp. 54-63. doi:10.1016/S0749-3797(00)00114-8

[4] A. P. Dignan, “A Decade of Experience of Examining Candidates for Entry to the Army," Journal of the Royal Army Medical Corps, Vol. 138, No. 1, 1992, pp. 19-22.

[5] C. A. McTimoney and L. J. Micheli, "Current Evaluation and Management of Spondylolysis and Spondylolisthesis,” Current Sports Medicine Reports, Vol. 2, No. 1, 2003, pp. 41-46.

[6] A. Saifuddin, J. White, S. Tucker and B. A. Taylor, "Orientation of Lumbar Pars Defects: Implications for Radiological Detection and Surgical Management," Journal of Bone and Joint Surgery. British Volume, Vol. 80, No. 2, 1998, pp. 208-211. doi:10.1302/0301-620X.80B2.8219

[7] C. J. Harvey, J. L. Richenberg, A. Saifuddin and R. L. Wolman, "Pictoral Review: The Radiological Investigation of Lumbar Spondylolysis," Clinical Radiology, Vol. 53, No. 10, 1998, pp. 723-728. doi:10.1016/S0009-9260(98)80313-9

[8] W. J. Beutler, B. E. Fredrickson, A. Murtland, C. A. Sweeney, W. D. Grant and D. Baker, "The Natural History of Spondylolysis and Spondylolisthesis: A 45-Year Follow-Up Evaluation,” Spine, Vol. 28, No. 10, 2003, pp. 1027-1035. doi:10.1097/01.BRS.0000061992.98108.A0

[9] Y. Weil, D. Weil, M. Donchin, G. Mann and A. Hashroni, "Correlation between Pre-Employment Screening X-Ray Findings of Spondylolysis and Sickness Absenteeism Due to Low Back Pain among Policemen of the Israeli Police Force,” Spine, Vol. 29, No. 19, 2004, pp. 2168-2172. doi:10.1097/01.brs.0000141171.81801.e5 
[10] E. Libson, R. A. Bloom and G. Dinari, "Symptomatic and Asymptomatic Spondylolysis and Spondylolisthesis in Young Adults,” International Orthopaedics, Vol. 6, No. 4, 1982, pp. 259-261.

[11] E. L. Steinberg, E. Luger, A. Arbel, A. Menachem and S. Dekel, “A Comparative Roentgenographic Analysis of the Lumbar Spine in Male Army Recruits with and without Lower Back Pain,” Clinical Radiology, Vol. 58, No. 12, 2003, pp. 985-989. doi:10.1016/S0009-9260(03)00296-4

[12] J. Iwamoto, H. Abe, Y. Tsukimura and K. Wakano, "Relationship between Radiographic Abnormalities of Lumbar Spine and Incidence of Low Back Pain in High School Rugby Players: A Prospective Study," Scandinavian Journal of Medicine \& Science in Sports, Vol. 15, No. 3, 2005, pp. 163-168.

doi:10.1111/j.1600-0838.2004.00414.x
[13] J. Iwamoto, H. Abe, Y. Tsukimura and K. Wakano, "Relationship between Radiographic Abnormalities of Lumbar Spine and Incidence of Low Back Pain in High School and College Football Players: A Prospective Study,” The American Journal of Sports Medicine, Vol. 32, No. 3, 2004, pp. 781-786. doi: $10.1177 / 0363546503261721$

[14] J. Hayton, "Reducing Medical Downgrading in a High Readiness Royal Marine Unit," Journal of the Royal Army Medical Corps, Vol. 150, No. 3, 2004, pp. 164-167.

[15] C. Milgrom, A. Finestone, B. Lev, M. Weiner and Y. Floman, "Overexertional Lumbar and Thoracic Back Pain among Recruits: A Prospective Study of Risk Factors and Treatment Regimens," Journal of Spinal Disorders \& Techniques, Vol. 6, No. 3, 1993, pp. 187-193. doi:10.1097/00002517-199306030-00001 